\title{
Parance Béatrice, De Saint Victor Jacques (dir.), Repenser les biens communs, Paris, CNRS éditions, 2014, 314 pages.
}

Bruno Villalba

\section{(2) OpenEdition Journals}

Electronic version

URL: http://journals.openedition.org/developpementdurable/11004

DOI: 10.4000/developpementdurable.11004

ISSN: $1772-9971$

Publisher

Association DD\&T

\section{Electronic reference}

Bruno Villalba, «Parance Béatrice, De Saint Victor Jacques (dir.), Repenser les biens communs, Paris, CNRS éditions, 2014, 314 pages. ", Développement durable et territoires [Online], Vol. 6, n² I Septembre 2015, Online since 30 September 2015, connection on 25 September 2020. URL : http:// journals.openedition.org/developpementdurable/11004; DOl : https://doi.org/10.4000/ developpementdurable.11004

This text was automatically generated on 25 September 2020 .

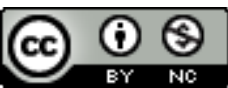

Développement Durable et Territoires est mis à disposition selon les termes de la licence Creative Commons Attribution - Pas d'Utilisation Commerciale 4.0 International. 


\section{Parance Béatrice, De Saint Victor Jacques (dir.), Repenser les biens} communs, Paris, CNRS éditions, 2014,

\section{4 pages.}

Bruno Villalba

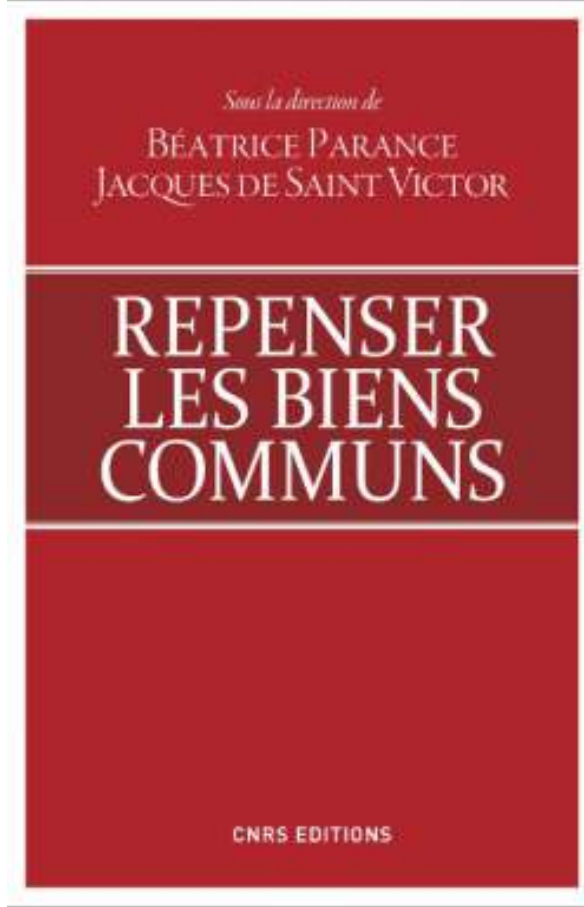

1 L'ouvrage collectif est animé par une réflexion centrale : comment penser la question de la propriété face à la transformation profonde des rapports économiques et de la situation environnementale dramatiquement frappée par des crises multiples? La 
réflexion est menée par deux juristes, Béatrice Parance (professeur de droit privé au laboratoire de Droit médical et de la santé de Paris $8^{1}$ ) et Jacques De Saint Victor (professeur d'histoire du droit au laboratoire Forces du droit de Paris 8), accompagné d'autres juristes, d'anthropologues, d'historiens et de philosophes ${ }^{2}$. Ils proposent un projet théorique ambitieux : procéder à une révolution juridique (notamment sur la question de la propriété) à partir de la notion de bien commun ${ }^{3}$, car elle interroge «le triptyque de la modernité juridique: Etat/marché/propriété» (p.22). Les auteurs contribuent ainsi, à partir d'une critique juridique, à l'élaboration d'une troisième voie, entre la privatisation et la nationalisation ${ }^{4}$.

Dans une introduction stimulante, les deux coordinateurs reviennent sur les évolutions principalement sociales et économiques (et trop peu écologiques) qui amènent à cette volonté de refondation du droit. Si la propriété a été l'élément constitutif du changement des sociétés modernes (principalement occidentales), elle se confronte désormais à une mutation des conditions de l'appropriation: les concentrations monopolistiques obligent à prendre en compte les conséquences de ce mécanisme d'usage exclusif des biens au profit de quelques sociétés. Cette "seconde révolution propriétaire» (p.10) aboutit à une vision trop extensive du droit de propriété. Cela conduit à s'interroger sur les conditions d'élaboration d'une autre forme de rapport pouvant exister entre les personnes et les choses. Loin de se cantonner à une critique de l'ordre néo-libéral 5 , le cadrage du livre insiste davantage sur l'importance de comprendre que nous passons d'un contexte historique "d'abondance et de progrès " à une société où nous devons penser notre rapport au monde en constant que les ressources sont limitées... : «nous sommes entrés dans des économies complexes, qui, dans certains domaines (énergétiques par exemple), s'apparentent de plus en plus à des économies de pénurie. » (p. 11)

Il convient donc d'élaborer d'autres modes plus inclusifs qu'exclusifs ${ }^{6} \mathrm{~d}^{\prime}$ appropriation. L'ouvrage récuse donc toute vision « radicale ", qu'elle soit inspirée par des approches collectivistes ou néo-libérale. Pour autant, son approche n'en est pas moins audacieuse, car il s'agit "sans remettre en cause le principe même de la liberté individuelle (et de son corollaire, la propriété privée individuelle), [de donner] la possibilité de réfléchir à la façon de gérer au mieux le rapport entre les hommes et les choses sans reproduire à l'identique le rapport individuel de possession car le droit libéral n'a pensé les traits absolus de la propriété qu'à partir de l'individu. » (pp. 20-21). L'introduction est l'occasion de rappeler combien interroger la propriété, aboutit à reconsidérer les principes de la souveraineté étatique, les principes des droits fondamentaux (la logique collective interroge le principe de l'individualisme juridique) etc.

Quatre parties structurent le déroulement de la démonstration. La première s'interroge sur « Comment appréhender les biens communs »? La deuxième se propose de traiter des " contours contemporains des "communs" ", tandis que la troisième aborde "Les logiques de protections». Enfin, la quatrième se centre sur «Les logiques d'accès». En tout, ce sont treize contributions, dont la conclusion, qui participent à la compréhension des enjeux théoriques du commun (juridique, anthropologique), à la délimitation des biens concernés (la biodiversité, le patrimoine, l'eau, l'internet) ou de certains acteurs plus spécifiquement (l'entreprise, l'Etat, l'individu). Les frontières entre ces différentes parties ne sont pas des plus intéressantes si l'on souhaite établir une interrogation transversale de l'enjeu du commun. Elles correspondent toutefois à la volonté de mieux défricher les conséquences d'une prise en considération de cette notion ${ }^{7}$. Notons que 
les différentes contributions ne participent pas toutes directement à l'intrigue présentée dans l'introduction.

On se doit de souligner la constante tentation de définir la « délicate» notion de bien commun $^{8}$. La définition a pour principal inconvénient de circonscrire un espace de réflexion et d'usage d'une notion, alors que la réflexion sur les conditions de production d'une définition nous renseigne beaucoup plus sur ce travail de sélection, de hiérarchisation et de structuration d'une notion. Comme le rappelle souvent les contributions, le commun est d'abord le résultat d'une construction sociale, historique et culturelle. A condition toutefois d'accepter le fait que le commun résulte alors d'une imposition normative, qui façonne ainsi des formes d'organisation sociale et conditionne ainsi notre rapport à l'environnement. Cette conflictualité dans l'imposition d'une perspective analytique et juridique est cependant souvent trop peu présente dans les contributions de cet ouvrage. Il s'agit là de la première limite à cet ouvrage. La prise en compte des processus de production de ce cadre normatif sociohistorique, porté par des rapports de force n'est pas suffisamment présente 9 .

Deuxième limite: la question écologique n'est pas suffisamment présente dans l'ouvrage. Elle est abordée à partir des enjeux socio-économiques qu'elle soulève (p. 48). Heureusement, la contribution de Béatrice Parance portant sur «La protection de la biodiversité. Entre protection et accès » (pp. 225-240) est vraiment très éclairante et permet de donner toute son importance à cet enjeu. Mais il est dommage que d'autres dimensions écologiques ne soient pas évoquées, car une meilleure prise en considération de cette question aurait sans doute pu contribuer à relativiser certaines options théoriques (l'idée que la rareté puisse être compensée par une meilleure gestion du droit de propriété par exemple), encore trop marquées par une perspective socio-technique. La conclusion rappelle pourtant utilement que les communs posent la question « des biens essentiels à la survie même de la vie humaine » (Michel Marzano, «Les "biens communs" : une alternative nouvelle?» p. 307.)

7 Ces deux remarques n'entachent aucunement la qualité réelle de l'ouvrage: les contributions permettent d'éclairer la richesse d'un débat juridique qui engage les catégories normatives des biens communs. On saisit alors qu'au-delà d'une simple discussion philosophique ou politique sur l'opportunité de développer ces biens communs, les juristes proposent déjà des modalités de leur transcription dans le droit positif.

\section{NOTES}

1. Voir son intervention sur https://www.youtube.com/watch?v=6wEuCmYNW-w

2. On regrettera, sur le plan formel, une absence de présentation détaillée des auteurs qui aurait permis de saisir comment la question des biens communs est abordée par les différentes disciplines juridiques.

3. L'usage constant des guillemets qui accompagnent cette notion, ainsi que les «biens communs » n'est cependant pas expliqué. 
4. Il s'agit là d'une perspective assez classique, qui constitue l'ossature de la démarche d'Elinor Ostrom, 2010, Gouvernance des biens communs. Pour une nouvelle approche des ressources naturelles, Bruxelle, De Boeck.

5. Il est à souligner combien le livre évite le piège d'une simple justification de la critique de cet ordre à partir d'une nouvelle interprétation des théories néo-marxistes (voir Noam Chomsky, 2014, Le bien commun, Paris, Ecosociété, Entretiens avec David Barsamian), ou bien encore, en invoquant simplement une revendication morale (comme au sein des courants altermondialistes, qui ont largement contribué à relégitimer cette notion de bien commun).

6. Issus du mouvement des enclosures qui étaient considérés par la pensée économique dominante comme une dynamique positive ayant engendré et constitué l'essor du capitalisme occidental.

7. Il aurait été intéressant de présenter, dans une courte introduction, les raisons expliquant ces subdivisions. Cela aurait permis de mieux appréhender les raisons des distinctions opérées.

8. "La tâche réservée aux juristes est très lourde. Car c'est à eux que revient la mission de trouver sinon une définition des " biens communs", tout au moins de leur attribuer une valeur normative. » p. 21. C'est aller un peu vite en besogne et c'est incontestablement sous-estimer le processus d'élaboration d'un cadre normatif qui n'appartient pas, fort heureusement, à une seule communauté.

9. Le livre de Bruno Boidin montre combien cette approche permet de saisir la difficulté d'élaboration d'un commun, alors qu'il est assujetti à des rapports de forces antagonistes, qui pourtant mobilisent tous la rhétorique du commun, Boidin B., La santé, bien public mondial ou bien marchand? Réflexions à partir des expériences africaines, Presses Universitaires du Septentrion, coll. Capitalisme, éthique, institutions, 2014.

\section{AUTHOR}

BRUNO VILLALBA

Bruno Villalba, Professeur de science politique, Agroparistech, CerapsFr 